\title{
Pushing the boundaries with collision collaboration: the marriage of ideas
}

\author{
Jeffrey C. Rathmell' and W. Kimryn Rathmell \\ 'Department of Pathology, Microbiology, and Immunology and 2Department of Medicine, Vanderbilt University Medical Center, Nashville, Tennessee, USA.
}

C ollaboration and interdisciplinary approaches in biomedical research are more prevalent than at any time in history due to the complexity of modern science and the rapid pace of discovery. The intersections of fields and the new concepts that emerge from approaching questions with different lenses can spur exciting discoveries. We discuss here the basic rubrics of collaboration and team-based science and the more complex intertwining of fields that can occur in multi-investigator projects, which we call collision collaboration. An added complexity emerges when the collaborating investigators are spouses, which we will examine separately. The fundamentals of communication, selfreflection, and engagement apply.

There is emerging literature on the critical aspects of successful teams in science (1), and there is little doubt that investigators will continue to evolve this space as increasingly large questions, as well as funding mechanisms that promote integrative concepts, drive the emergence of more collaborative strategies. Certainly, a perspective piece written in 2020 has to acknowledge the tremendous value in collaborative efforts to contain SARS-Cov2. Collaborative groups and consortia from around the world have made tremendous gains in determining the epidemiology, spread, and natural history of this disease, and thanks to team science approaches, a growing list of therapeutic agents have been evaluated for efficacy and rapidly advanced to clinical care. The response has been nothing short of a "call to arms," as referred to in the virtual address to the membership of the Association of American Physicians (2). A key component to the rapid expansion of collaborative teams has been the availability of technologies that allow virtual collaborations. Crites et. al. describe four models of virtual collaboration that provide the structure necessary for recognizing the needs, expectations, and potential outcomes for the type of team being created (3).

While productive collaborations take many forms, couples are common in academic institutions and this perspective tackles the unique collaboration that includes household engagement. In a sense, our collaboration began in the autumn of 1987, in freshman biology at the University of Northern Iowa. As we write this, we are coming up on 30 years married, having spent those years with marriage, life, and science intertwined. We each started independent research careers at different institutions with distinct areas of focus. Although our research fields of immunology, metabolism, and cancer biology were clearly related, we barely collaborated scientifically over our first decade of independence and only began in earnest when we made a move that brought us to the same institution. Those initial years were important for us each to establish our niches and distinctive expertise, but the integration of those experiences is now opening new and exciting doors. This perspective serves three purposes: first, to lend our thoughts on the merits of collaborative science; second, to provide a commentary on the growing phenotype of two-career scientist couples; and third, to highlight themes that penetrate both of these collaborations that we will enumerate.

\section{The necessity of team science and collaboration}

Why engage in team science? Team science is not right for every project, and just
Conflict of interest: JCR and WKR declare research support from Incyte. JCR declares research support from Sitryx Therapeutics. WKR declares clinical trial support from Pfizer, Roche, Calithera, Merck/Peloton, Bristol Myers Squibb, and Incyte.

Copyright: () 2021, American Society for Clinical Investigation.

Reference information: J Clin Invest. 2021;131(2):e145964. https://doi.org/10.1172/JCl145964. as the assay has to be selected to address the question, the format of the research platform (which we have labeled unit, collaborative, team science, or collision) has to be selected to meet the needs of the project.

Scientific investigation is intensely personal, and the questions that are produced in pushing or breaking through a boundary are traditionally individualistic due to the effort of mentally working through a set of ideas. A project team then becomes aligned through the focused effort into what we here call a unit.

Of course, collaboration is a longstanding tenet of successful research advances. Working with fellow scientists to gain insights on an idea, pathway, or process, to acquire a necessary reagent or skill, or to apply something new to a line of investigation can be essential to completing a story. It is increasingly common for manuscripts to include authors from multiple independent laboratories. Our early collaborative manuscripts underscore this concept. We had a few overlapping areas and found ourselves on each other's papers occasionally. These kinds of collaborations for an assay, tissue source, or reagent that the other group possesses are transactional and retain the identity of each investigative unit.

There are times when science is too big and too complicated for any one person or group to be expert in everything. Projects such as the Cancer Genome Atlas that require experts in tissue pathology, data curation, a variety of -omics, and content in each disease to work together, or the challenge of pandemic viruses is a great example. These projects are too massive and too multifaceted for any one group to manage alone. The keys to these collaborations, which we call "multidisciplinary," are excellent project management and having well-defined goals so that each component stays on track. This brand of investigation is most 


\section{Table 1. Pearls of wisdom for successful collaborations}

Communicate effectively and often, and in person.

Bring your whole self, unique background, and expertise to the collaboration.

Share ideas, even in their rawest form. Be honest in providing feedback.

Expect to hear unexpected feedback; welcome it at face value.

Embrace the challenges of breaking into staunchly held field beliefs.

Learn about the other field and develop a comfort level with the data and vocabulary.

Engage the people doing the work to collaborate directly.

Enjoy getting to know your colleagues research team. Invest in the people doing the work.

Make time for face-to-face interaction and celebrate wins together. Have fun!

emblematic of a team, with individual units playing a defined role to make the team successful.

A further version of team science occurs when the teams merge effectively and blur the independent unit definitions. This is perhaps where team science can be the most challenging and intimidating. It is one thing to trust a collaborator with unique content expertise. It is another to allow another group to develop the research direction with you. Many tremendous examples of hybrid, integrated, or communal research groups have advanced breakthroughs in human medical science throughout history. We call this "collision collaboration."

\section{Collision collaboration}

The collaboration that our labs have found ourselves in recently aligns with the theme of collision collaboration. Two expert groups with independent directions merge their ideas, vocabulary, and conceptual frameworks to work in what becomes a completely new space. Our labs had developed longstanding independent lines of investigation in renal cell carcinoma molecular biology and immunometabolism, respectively, and we now find our groups have naturally coalesced around a range of shared and complementary interests.

What brought us together to merge these two fields? Some of it was probably unavoidable. As is the case for many science couples, we talk about our science all day together. We read each other's grants and papers. We are deeply aware of what each other's labs are doing, so it was almost inevitable that we would find an exciting area of overlap. As well, the merger of immunology with renal cell carcinoma was brought to bear as the first checkpoint inhibitors were emerging with activity in renal cell carcinoma as one of the early disease types to demonstrate activity. The opportunities in that space became immediately apparent.

Critical to the success of our current collaboration, however, was that our lab personnel also saw the value in working in this new space of kidney cancer immune metabolism. Our change of institutions was a pivot point because, by necessity, we merged our lab meetings and now many lab members are jointly mentored. That tradition continues today, with a weekly meeting where everyone presents, whether working in the mainstream of one of the labs or wading into this new hybrid workspace. We also have other individuals and groups that ask to join our meetings and further diversify the scientific discourse and enrich the growing collision. This kind of collaboration carries the benefit of exciting explosive new science brainstorming, the "Aha" moments when you find a core concept in one field is a neglected topic area in the other and the intensely satisfying discovery mode of peering into a new area - in our case, into the tumor microenvironment.

Challenges can also exist in navigating these dual projects. Effective communication is key. Regularly assessing the status of projects and ensuring that all parties are aligned is essential. This becomes a challenge when assigning funding and, in particular, in assigning authorship and authorship order. A focus for our field needs to be in providing new conventions that equally reward investigators who engage in collaborative research. The limitation of significant contributions being relegated to first and last author position lacks the granularity and sophistication for recognizing true measures of effort.

\section{On the topic of spousal collaboration}

As increasing numbers of women are pursuing careers in science, it is not surprising to see the number of science career couples grow. Due to the timing and duration of training, having a life partner in the same general field is common. This is hardly a new concept, and notable couples in the sciences include Marie and Pierre Curie and Gerti and Carl Cori, both Nobel Prize winning couples. There can be incredible power behind putting two minds to a task and doing so in the laboratory and at the dinner table. This form of collaboration also exists on a spectrum, from fully dual laboratories, where both partners focus on a singular theme, to situations more like ours where each holds a fully independent laboratory, but there are opportunities to collaborate intensely.

Let's be frank. Couples who pursue wholly combined research programs face unique sets of challenges in maintaining dual identities. The benefits of shared equipment and space, jointly mentored lab members, and the deep focus on a common research theme are real, in addition to some greater lab stability and culture. However, as much as science is the great equalizer, quantitative, and rigorous in assigning credit, there remains gender bias. Further, unequal professional career levels may influence how roles and contributions are viewed (4).

From our perspective, it was valuable for our early individual careers that we very visibly pursued independent lines of investigation. Our times at the different (and athletic rival) institutions of Duke University and University of North Carolina were critical to developing our identities with different groups of researchers who knew us not as partners but as individuals with distinct scientific interests. It is only recently that we find ourselves attending the same meetings. And it is refreshing to meet new colleagues who have been longterm associates of the other and to rapidly expand our networks as we move into this new arena for both of us.

Not to be dismissed, however, is the advantage of the daily discussions, of having a critical grant and paper reviewer in your house, of having a sounding board as you navigate reviewer comments, or of having a partner who understands the 
oddities and cyclic nature of academic research. Our experience is that the emerging trend of career science couples will only heighten the pace and level of research emerging across the board.

\section{Rathmell tips for successful collaborative science}

Both of us have similar collaborations with other investigators that pursue blended themes of research, represented by our proclivity to write multiple-PI grants. These pearls (Table 1) stem from nearly 20 years of managing effective collaborations, some that are transient and others that are longstanding.

Fundamental project management, role definition, and frequent communication are essential to successful col- laborations. Practice in active listening, model building and rebuilding, and transparency in data sharing is also necessary. Dealing with uncertainty and trusting in an unknown outcome can be barriers. Perhaps most challenging is the need to compromise - not in standards, but in pacing, direction, and recognition. Ultimately, the level of honesty, trust, and transparency that is needed to navigate a successful collaboration is in some ways ideally modeled by the partnership that is displayed by couples.

In summary, our perspective is that collaborative science, as we have experienced it between our two groups and as we participate in it with other researchers, has the potential to dramatically expand horizons, to accelerate findings, and to provide an added level of enjoyment to a career working in discovery.

Address correspondence to: W. Kimryn Rathmell, 1161 21st St., Medical Center North, Suite D3100, Nashville, Tennessee 37232, USA. Phone: 615.343.8701 Email: kimryn.rathmell@vumc.org

1. Bennett LM, Gadlin H. Collaboration and team science: from theory to practice. J Investig Med. 2012;60(5):768-775.

2. Klotman ME. Call to arms during disruption. J Clin Invest. 2020;130(6):2729-2730.

3. Crites GE, Berry A, Hall E, Kay D, Khalil MK, Hurtubise L. Applying multiple frameworks to establish effective virtual collaborative teams in academia: a review and recommendations. Med Educ Online. 2020;25(1):1742968.

4. Helena M. Pycior, et al, eds. Creative Couples In The Sciences. Rutgers University Press; 1996. 